\title{
Two-dimensional ferromagnet/semiconductor transition metal dichalcogenide contacts: p-type Schottky barrier and spin-injection control
}

\author{
Li-Yong Gan, Qingyun Zhang, Yingchun Cheng, ${ }^{*}$ and Udo Schwingenschlögl" \\ PSE Division, KAUST, Thuwal 23955-6900, Kingdom of Saudi Arabia
}

(Received 26 September 2013; published 18 December 2013)

\begin{abstract}
We study the ferromagnet/semiconductor contacts formed by transition metal dichalcogenide monolayers, focusing on semiconducting $\mathrm{MoS}_{2}$ and $\mathrm{WS}_{2}$ and ferromagnetic $\mathrm{VS}_{2}$. We investigate the degree of $p$-type doping and demonstrate tuning of the Schottky barrier height by vertical compressive pressure. An analytical model is presented for the barrier heights that accurately describes the numerical findings and is expected to be of general validity for all transition metal dichalcogenide metal/semiconductor contacts. Furthermore, magnetic proximity effects induce a $100 \%$ spin polarization at the Fermi level in the semiconductor where the spin splitting increases up to $0.70 \mathrm{eV}$ for increasing pressure.
\end{abstract}

DOI: $10.1103 /$ PhysRevB.88.235310

PACS number(s): 73.22.-f, 73.21.Ac, 73.30.+y

\section{INTRODUCTION}

Two-dimensional (2D) materials exhibit unique properties, which often are distinctly different from their bulk counterparts, thus having created tremendous interest in synthesis and application. ${ }^{1-3}$ In particular, the monolayers of semiconducting transition metal dichalcogenides (TMDCs), such as $\mathrm{MoS}_{2}$ and $\mathrm{WS}_{2}$, exhibit versatile electronic, ${ }^{4-8}$ optical, ${ }^{9-12}$ mechanical, ${ }^{13-15}$ and chemical $^{16-18}$ properties, offering opportunities beyond the more famous graphene and therefore opening up new fundamental as well as technological avenues for inorganic 2D materials in a range of fields, including electronic devices, catalysis, and energy storage. ${ }^{19}$ Semiconductor-to-metal transitions have been predicted for TMDC bilayers in external electric fields ${ }^{20}$ and under vertical compressive pressure. ${ }^{21}$ In order to tune the electronic properties of semiconducting 2D TMDCs, hybrid systems have been put forward. Radisavljevic and co-workers ${ }^{22}$ have reported on few-layer 2D TMDC integrated electronic circuits that are able to perform digital logic operations. The $\mathrm{MoS}_{2}$ /graphene hybrid system has been reported to show a high electron conductivity and excellent electrochemical and thermoelectric performances. $^{23-26}$ Therefore, combining TMDCs with other $2 \mathrm{D}$ layered materials is a viable and promising way to realize vertical heterostructures and hybrid all-2D devices. ${ }^{27,28}$

Metal/semiconductor contacts play a key role in modern electronic and photonic devices, since the created Schottky barriers dominate the transport behavior. ${ }^{29}$ It has been found that the barrier height in $\mathrm{MoS}_{2}$ drastically depends on the electrodes. ${ }^{30}$ More recently, a back gate voltage has been applied to manipulate the barrier height in $\mathrm{MoS}_{2}$ field effective transistors contacted with cobalt electrodes. ${ }^{31}$ In addition, for potential spintronics applications, understanding the contacts with ferromagnetic substrates is crucial to realize efficient spin injection and enhance the spin polarization of the current across the interface. ${ }^{32,33}$ Introduction of transition metal atoms $^{34}$ and contacting with ferromagnetic substrates ${ }^{31}$ have been reported to yield spin injection and spin transport in monolayer $\mathrm{MoS}_{2}$. 2D TMDCs constitute a large family of materials and possess the advantage that the electronic properties range from ferromagnets (such as $\mathrm{VS}_{2}$ and $\left.\mathrm{NbS}_{2}\right)^{35-38}$ to semiconductors, ${ }^{39}$ opening the possibility of ferromagnet/semiconductor contacts for engineering all-2D electronic devices and for realizing spin polarization. ${ }^{28}$ In this context, we propose a class of ferromagnet/semiconductor contacts completely based on TMDC monolayers.

In the following, we investigate Schottky contacts in 2D TMDCs, focusing on the nonmagnetic semiconductors $\mathrm{MoS}_{2}$ and $\mathrm{WS}_{2}$ and the ferromagnet $\mathrm{VS}_{2}$ as prototypes. We will argue that the degree of $p$-type doping and the corresponding Schottky barrier heights in $\mathrm{MoS}_{2}$ and $\mathrm{WS}_{2}$ can be controlled by vertical compressive pressure. A comprehensive picture is derived to understand the mechanism of the pressure dependence. We will also argue that magnetic proximity effects $^{40-42}$ induce $100 \%$ spin polarization in $\mathrm{MoS}_{2}$ and $\mathrm{WS}_{2}$ at the Fermi level $\left(E_{F}\right)$ in a wide pressure range. These findings pave the way for designing coherent dopant-free all-2D contacts for future spin transport applications.

\section{COMPUTATIONAL METHODS}

First-principles calculations are performed using the Vienna Ab Initio Simulation Package with the spin polarized Perdew, Burke, and Ernzerhof generalized gradient approximation functional. In each case we apply a vacuum slab of at least $29 \AA$ thickness. A cutoff energy of $500 \mathrm{eV}$ and a $\Gamma$-centered $30 \times 30 \times 1 k$-mesh are used. The geometry is optimized until all residual forces are less than $0.01 \mathrm{eV} / \AA$. Because of the absence of strong bonding a damped van der Waals correction, ${ }^{43,44}$ which is a significant improvement with respect to the generalized gradient approximation (though dipole-dipole corrections are limited in precision), is adapted to model nonbonding forces. We note that the Schottky barrier is an intrinsic property of the interface and defined by the relative alignment of $E_{F}$ of the metal and the valence-band maximum (VBM, $p$-type barrier height $\Phi_{\mathrm{B}, \mathrm{p}}$ ) or conductionband minimum (CBM, n-type barrier height $\Phi_{\mathrm{B}, \mathrm{n}}$ ) of the semiconductor.

\section{RESULTS AND DISCUSSION}

$\mathrm{VS}_{2}$ monolayers exist in two polymorphs, trigonal prismatic $\left(h \mathrm{VS}_{2}\right)$ and octahedral $\left(t \mathrm{VS}_{2}\right)$, with $D_{3 h}$ and $D_{3 d}$ point groups, respectively. ${ }^{39}$ Monolayer $\mathrm{VS}_{2}$ has the former structure at and below room temperature. ${ }^{45}$ The properties obtained in our calculations for the two phases are 
TABLE I. Total energy ( $E_{\text {tot }}$, normalized), energy difference $(\Delta E)$ between spin degeneracy and polarization, in-plane lattice constant $(a)$, V-S bond length $\left(d_{\mathrm{V}-\mathrm{S}}\right)$, layer thickness $\left(d_{\mathrm{S}-\mathrm{S}}\right)$, magnetic moment $(M)$, and work function $(\Phi)$ in the trigonal prismatic $\left(h \mathrm{VS}_{2}\right)$ and octahedral $\left(t \mathrm{VS}_{2}\right)$ phases.

\begin{tabular}{lccccccc}
\hline \hline & $\begin{array}{c}E_{\text {tot }} \\
(\mathrm{meV})\end{array}$ & $\begin{array}{c}\Delta E \\
(\mathrm{meV})\end{array}$ & $a(\AA)$ & $d_{\mathrm{V}-\mathrm{S}}(\AA)$ & $d_{\mathrm{S}-\mathrm{S}}(\AA)$ & $M\left(\mu_{\mathrm{B}}\right)$ & $\Phi(\mathrm{eV})$ \\
\hline$h \mathrm{VS}_{2}$ & 0 & 48 & 3.174 & 2.362 & 2.981 & 1.00 & 5.87 \\
$t \mathrm{VS}_{2}$ & 27 & 12 & 3.174 & 2.349 & 2.940 & 0.48 & 5.41 \\
\hline \hline
\end{tabular}

summarized in Table I. The former is $27 \mathrm{meV}$ more stable, with the energy difference between spin degeneracy and polarization four times that of the latter. The in-plane lattice constants are the same, whereas the V-S bonds are slightly longer in $h \mathrm{VS}_{2}$. The calculated band structures, see Fig. 1, indicate that both phases are metallic with magnetic moments of $1.00 \mu_{B}$ and $0.48 \mu_{B}$ per unit cell. In $h \mathrm{VS}_{2}$ the magnetic moments of $\mathrm{V}$ and $\mathrm{S}$ are 1.02 and $-0.05 \mu_{B}$, respectively, while the corresponding values in $t \mathrm{VS}_{2}$ are 0.51 and $-0.03 \mu_{B}$. The calculated work function of $h \mathrm{VS}_{2}$ is $0.46 \mathrm{eV}$ larger than that of $t \mathrm{VS}_{2}$. All these results agree well with previous experimental and theoretical studies. ${ }^{35,37,46}$

The relaxed lattice constant of both $\mathrm{MoS}_{2}$ and $\mathrm{WS}_{2}$ is $3.181 \AA$, i.e., the lattice mismatch to $\mathrm{VS}_{2}$ is tiny $(\sim 0.2 \%)$. Thus, the average value of $3.177 \AA$ is adopted for all the hybrid systems. For the contact with trigonal prismatic $h \mathrm{VS}_{2}$ two stacking modes are considered, namely $\mathrm{AA}$ and $\mathrm{AB}$, in which the Mo or $\mathrm{W}$ atoms are on top of $\mathrm{V}$ and $\mathrm{S}$, respectively. Correspondingly, the interfaces are labeled $h \mathrm{VS}_{2} / \mathrm{MoS}_{2}-\mathrm{AA}$, $h \mathrm{VS}_{2} / \mathrm{MoS}_{2}-\mathrm{AB}, h \mathrm{VS}_{2} / \mathrm{WS}_{2}-\mathrm{AA}$, and $h \mathrm{VS}_{2} / \mathrm{WS}_{2}-\mathrm{AB}$. For the contact with octahedral $t \mathrm{VS}_{2}$ there are in total six configurations: The atop-I and II patterns have $S$ above $S(1)$ and Mo or W above $\mathrm{V}$ and $\mathrm{S}(2)$, respectively, the hcp-I and II patterns have $S$ above $V$ and Mo or $W$ above $S(1)$ and $S(2)$, and the fcc-I and II patterns have $S$ above $S(2)$ and Mo or $\mathrm{W}$ above $\mathrm{S}(1)$ and $\mathrm{V}$. The structures of the $h \mathrm{VS}_{2} / \mathrm{MoS}_{2}$ and $t \mathrm{VS}_{2} / \mathrm{MoS}_{2}$ interfaces are shown in Fig. 2(d). The binding energy of an interface is defined as $E_{B}=E_{\mathrm{IS}}-E_{M}-E_{V}$, where $E_{\mathrm{IS}}, E_{M}$, and $E_{V}$ represent the total energies of the hybrid system, $\mathrm{MoS}_{2}$ or $\mathrm{WS}_{2}$, and $\mathrm{VS}_{2}$, respectively. The obtained binding energies per interface metal atom as a function of the interface separation $D$ between $\mathrm{MoS}_{2}$ or $\mathrm{WS}_{2}$ and $\mathrm{VS}_{2}$ are shown in Figs. 2(a)-2(c).

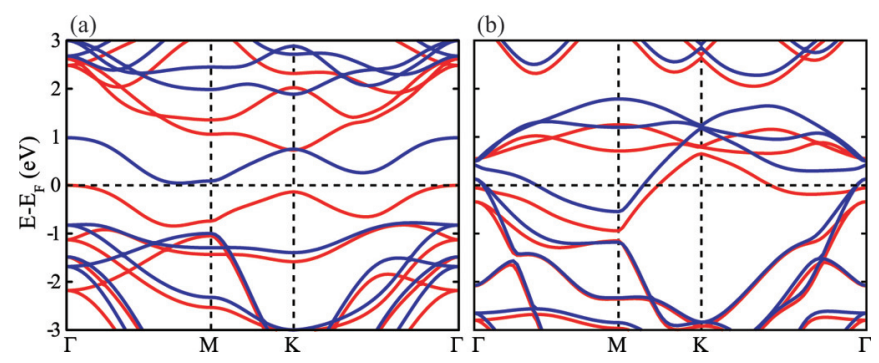

FIG. 1. (Color online) Spin polarized band structures of (a) $h \mathrm{VS}_{2}$ and (b) $t \mathrm{VS}_{2}$. Red and blue lines correspond to the spin majority and minority bands, respectively.
We first focus on the bilayer systems with $\mathrm{MoS}_{2}$ or $\mathrm{WS}_{2}$ on $\mathrm{VS}_{2}$ in the trigonal prismatic phase. As is shown in Figs. 2(a) and 2(b), the interaction between $\mathrm{MoS}_{2}$ or $\mathrm{WS}_{2}$ and $\mathrm{VS}_{2}$ is similar to that in $\mathrm{MoS}_{2} / \mathrm{Ti}_{2} \mathrm{CY}_{2},-0.14 \mathrm{eV}$ as compared to $-0.13 \mathrm{eV}$ at the respective equilibrium position. ${ }^{47}$ Due to the larger S-S repulsion, the AA stacking is energetically less favorable in both $h \mathrm{VS}_{2} / \mathrm{MoS}_{2}$ and $h \mathrm{VS}_{2} / \mathrm{WS}_{2}$. The equilibrium positions are the same in the two systems, 3.7 and $3.1 \AA$ in the AA and AB stackings, respectively. For a separation larger than $\sim 3.9 \AA$ the $\mathrm{AA}$ and $\mathrm{AB}$ energy profiles overlap, indicating that the cases are degenerate with the interface formation still being exothermic. Figure 3 shows densities of states of $\mathrm{MoS}_{2}, \mathrm{WS}_{2}$, and $\mathrm{VS}_{2}$ in $h \mathrm{VS}_{2} / \mathrm{MoS}_{2}-\mathrm{AA}$ and $h \mathrm{VS}_{2} / \mathrm{WS}_{2}-\mathrm{AA}$ at $D=2.4 \AA$ and at the equilibrium positions to explore the electronic structure variations in the semiconductors upon the interface formation. It can be seen that the interface interaction modifies the electronic properties remarkably. At $D=2.4 \AA$ distinct orbital overlaps between $\mathrm{MoS}_{2}$ or $\mathrm{WS}_{2}$ and $\mathrm{VS}_{2}$ can be seen near $E_{F}$, suggesting strong hybridization. The induced states ${ }^{48}$ near $E_{F}$ suggest that the metallic character dominates the semiconducting natures of $\mathrm{MoS}_{2}$ and $\mathrm{WS}_{2}$. The metallic features are different from those in semiconducting TMDCs bilayers, where they occur as upshift of the VBM at the $\Gamma$ point and downshift of the $\mathrm{CBM}$ at the $K$ point for decreasing interlayer separation. ${ }^{21}$ Additionally, evident spin polarization appears in both $\mathrm{MoS}_{2}$ and $\mathrm{WS}_{2}$ due to magnetic proximity effects. ${ }^{40-42}$ Because the effective $S-S$ distance is much smaller in the AA stacking, a lower pressure threshold has been found for the semiconductor-to-metal transition in Ref. 21. It can be expected that similar metallic features in $\mathrm{MoS}_{2}$ and $\mathrm{WS}_{2}$ can be obtained in the $\mathrm{AB}$ stacking at high enough pressure. At the respective equilibrium positions in $h \mathrm{VS}_{2} / \mathrm{MoS}_{2}$-AA and $h \mathrm{VS}_{2} / \mathrm{WS}_{2}$-AA the density of states shows that both $\mathrm{MoS}_{2}$ and $\mathrm{WS}_{2}$ preserve their semiconducting characters with slightly smaller band gaps of 73 and $41 \mathrm{meV}$, respectively. Moreover, it can be seen from Figs. 3(b) and 3(d) that $E_{F}$ is close to the VBM, indicating a strong $p$-type doping with barrier heights of 0.26 and $0.20 \mathrm{eV}$.

According to Figs. 2(a) and 2(b), it is much more likely to have $\mathrm{AB}$ stacking in $h \mathrm{VS}_{2} / \mathrm{MoS}_{2}$ and $h \mathrm{VS}_{2} / \mathrm{WS}_{2}$ interfaces for a separation less than $3.1 \AA$. Electronic band structures for AB stacking at $D=2.4 \AA$ and at the equilibrium positions are given in Figs. 4(a) and 4(b). Due to the similarity between $h \mathrm{VS}_{2} / \mathrm{MoS}_{2}-\mathrm{AB}$ and $h \mathrm{VS}_{2} / \mathrm{WS}_{2}-\mathrm{AB}$ we only show the former case. At $D=2.4 \AA$ the $\mathrm{MoS}_{2} \mathrm{VBM}$ at the $\Gamma$ point is lifted higher than that at the $K$ point due to the typical interface interaction, ${ }^{21,49}$ and the band gap is narrowed by $0.49 \mathrm{eV}$. Additionally, the VBM at the $\Gamma$ point shows an evident spin splitting of $0.34 \mathrm{eV}$ with one spin channel even crossing $E_{F}$, suggesting a shallow $p$-type doping. In the case of the equilibrium separation the VBM is still located at the $\Gamma$ point, while the band-gap reduction $(0.16 \mathrm{eV})$ and spin splitting $(0.11 \mathrm{eV})$ are less pronounced. The VBM in both spin channels is located below $E_{F}$, giving rise to a $p$-type Schottky barrier of $0.20 \mathrm{eV}$. The trend of the band-gap variation and $\Gamma$ point spin splitting for $D=2.4$ to $3.1 \AA$ in Fig. 4(d) shows that $h \mathrm{VS}_{2} / \mathrm{MoS}_{2}-\mathrm{AB}$ and $h \mathrm{VS}_{2} / \mathrm{WS}_{2}-\mathrm{AB}$ behave similarly: When the separation decreases the band gap decreases and the spin splitting increases monotonously. It can be deduced that further reduction of the interface separation will result in shallower 
(a)

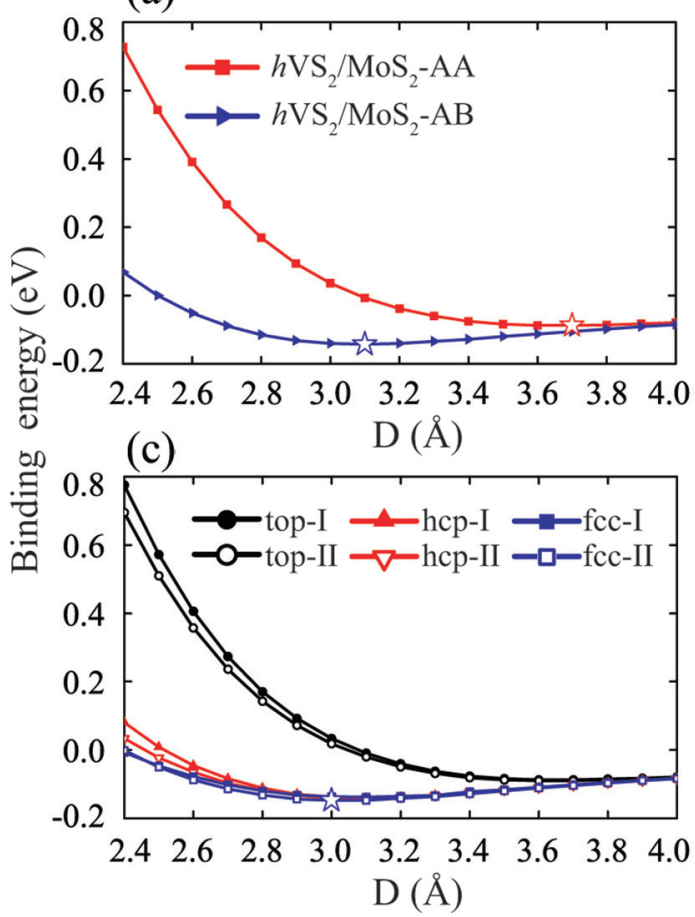

(b)

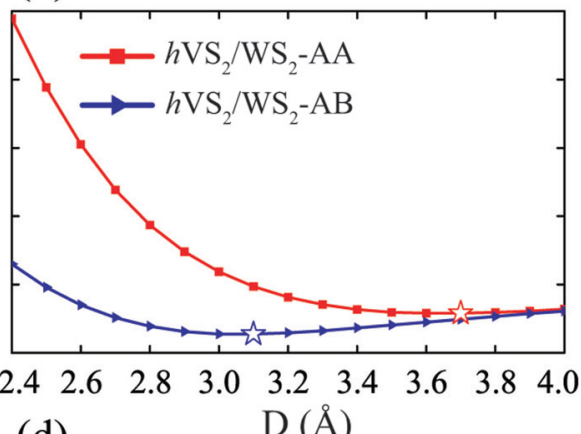

(d)

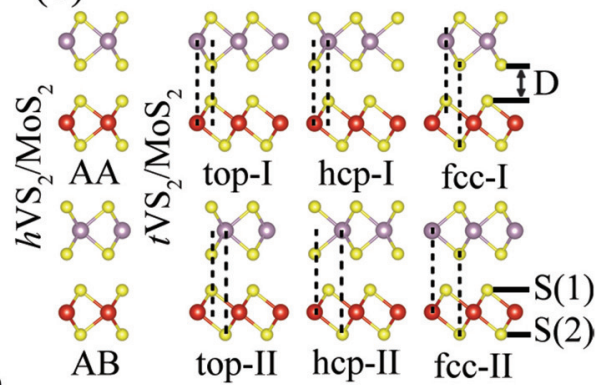

FIG. 2. (Color online) Binding energy per interface metal atom as a function of the interface separation $(D)$ in $\left(\right.$ a) $h \mathrm{VS}_{2} / \mathrm{MoS}_{2},(\mathrm{~b})$ $h \mathrm{VS}_{2} / \mathrm{WS}_{2}$, and (c) $t \mathrm{VS}_{2} / \mathrm{MoS}_{2}$. The equilibrium positions are indicated by stars. (d) Side views of the nonequivalent configurations of $h \mathrm{VS}_{2} / \mathrm{MoS}_{2}$ and $t \mathrm{VS}_{2} / \mathrm{MoS}_{2}$. Yellow, gray, and red balls represent $\mathrm{S}, \mathrm{Mo}$, and $\mathrm{V}$ atoms, respectively.

$p$-type doping and in an enhanced spin splitting in the two semiconductors.

We turn to the interfaces with $\mathrm{VS}_{2}$ in the octahedral phase. As discussed above, $\mathrm{MoS}_{2}$ and $\mathrm{WS}_{2}$ in contact with $h \mathrm{VS}_{2}$ show very similar results. Thus, we only consider the $t \mathrm{VS}_{2} / \mathrm{MoS}_{2}$ interface as an example. Figure 2(c) shows the binding energies as a function of the interface separation for the six configurations; see columns 2 to 4 in Fig. 2(d). According to the results, the six cases can be divided into two

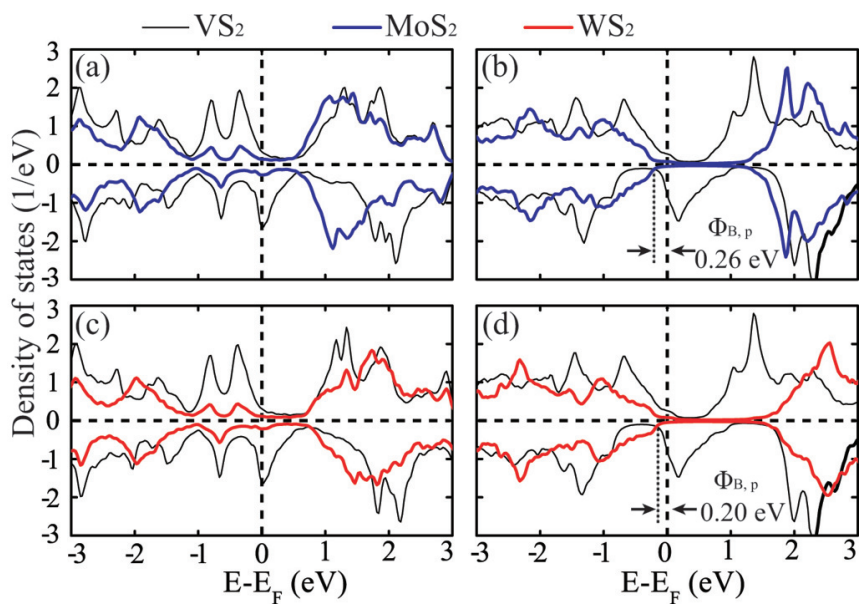

FIG. 3. (Color online) Spin polarized partial density of states for $\mathrm{VS}_{2}, \mathrm{MoS}_{2}$, and $\mathrm{WS}_{2}$ in $h \mathrm{VS}_{2} / \mathrm{MoS}_{2}-\mathrm{AA}$ and $h \mathrm{VS}_{2} / \mathrm{WS}_{2}-\mathrm{AA}$ for (a), (c) an interface separation of $2.4 \AA$ and (b), (d) the equilibrium position. In (b) and (d) the $p$-type Schottky barrier heights $\left(\Phi_{\mathrm{B}, \mathrm{p}}\right)$ are indicated. groups, similar to $h \mathrm{VS}_{2} / \mathrm{MoS}_{2}$ : Arrangements with the $\mathrm{S}$ atom in $\mathrm{MoS}_{2}$ on top of $\mathrm{S}(1)$ in $t \mathrm{VS}_{2}$, namely $t \mathrm{VS}_{2} / \mathrm{MoS}_{2}$-top-I and II, are less stable and belong to the AA stacking class, while the other four types are similar to the $\mathrm{AB}$ stacking and are more likely to form. Specifically, the four cases are almost degenerate, with fcc-II being slightly more stable, suggesting that all can be fabricated. The band structure of the fcc-II
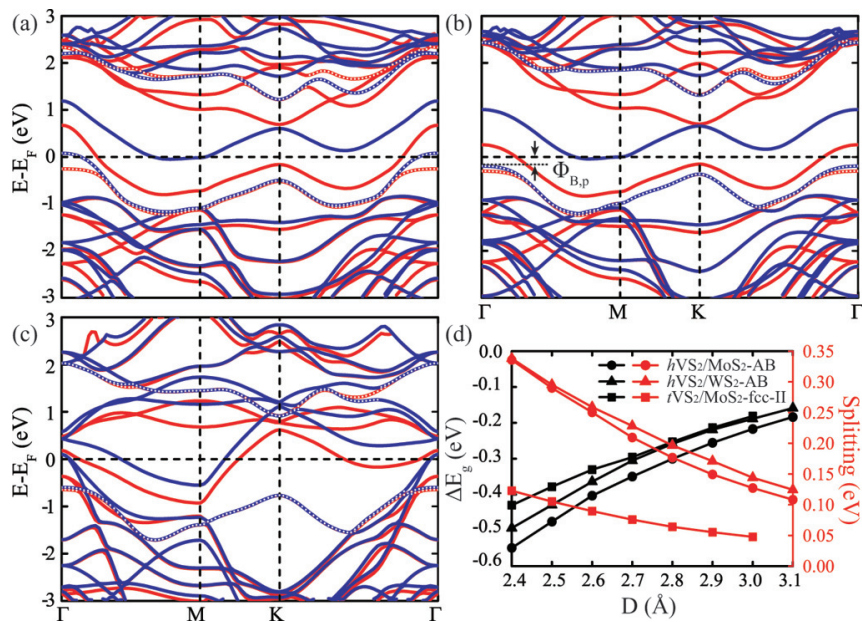

FIG. 4. (Color online) Spin polarized band structures of $h \mathrm{VS}_{2} / \mathrm{MoS}_{2}-\mathrm{AB}$ for (a) $D=2.4 \AA$, (b) the equilibrium position, and (c) $t \mathrm{VS}_{2} / \mathrm{MoS}_{2}$-fcc-II at the equilibrium position. The $\mathrm{MoS}_{2}$ derived conduction and valence bands in the hybrid systems are indicated by white dotted curves. (d) Band-gap variation (black) and spin splitting at the $\Gamma$ point (red) of $\mathrm{MoS}_{2}$ and $\mathrm{WS}_{2}$ as a function of the interface separation from $D=2.4 \AA$ to the respective equilibrium position. 
configuration at the equilibrium separation of $3.0 \AA$ is shown in Fig. 4(c) to investigate the electronic structure variations of $\mathrm{MoS}_{2}$ in contact with $t \mathrm{VS}_{2}$. The $\mathrm{VBM}$ of $\mathrm{MoS}_{2}$ is located at the $\Gamma$ point with the band gap narrowed by $0.18 \mathrm{eV}$ and displays spin splitting. Moreover, $E_{F}$ is located only $0.16 \mathrm{eV}$ below the midgap $(\mathrm{CBM}+\mathrm{VBM}) / 2$, suggesting rather weak $p$-type doping. The corresponding Schottky barrier height is calculated to be $0.60 \mathrm{eV}$. Figure 4(d) shows as a function of the interface separation the $\mathrm{MoS}_{2}$ band-gap variation and VBM spin splitting in $t \mathrm{VS}_{2} / \mathrm{MoS}_{2}$-fcc-II. Similar to the trends in $h \mathrm{VS}_{2} / \mathrm{MoS}_{2}-\mathrm{AB}$, the band gap increases and the spin splitting decreases monotonously as the separation increases, with the band-gap changes being comparable in $h \mathrm{VS}_{2} / \mathrm{MoS}_{2}$ and $h \mathrm{VS}_{2} / \mathrm{WS}_{2}$. However, a much smaller spin splitting is found in $t \mathrm{VS}_{2} / \mathrm{MoS}_{2}$-fcc-II due to the smaller magnetic moment of $t \mathrm{VS}_{2}$.

It has been demonstrated above that different Schottky barrier heights are obtained by adjusting the interface separation in contact with both $h \mathrm{VS}_{2}$ and $t \mathrm{VS}_{2}$. In order to access the controllability of the barrier height in the experiment, the interface separations are next converted into vertical compressive pressures $(P)$, which are calculated from the energy cost per unit area for reducing the separation,

$$
P=\frac{E-E_{0}}{\left(D_{0}-D\right) A},
$$

where $E$ and $E_{0}$ are the total energies at interface separations of $D$ and $D_{0}$ (equilibrium), and $A$ is the area of the cell. Note that the pressure induced volume change is essentially due to the interface spacing, while only tiny shifts of the atomic positions are observed. The obtained barrier height as a function of $P$ in $h \mathrm{VS}_{2} / \mathrm{MoS}_{2}-\mathrm{AB}$ (circles) and $t \mathrm{VS}_{2} / \mathrm{MoS}_{2}$-fcc-II (squares) is displayed in Fig. 6(b). At $P=4.3(D=2.5 \AA)$ and $5.5 \mathrm{GPa}$ ( $D=2.4 \AA$ ) the $\mathrm{VBM}$ of $\mathrm{MoS}_{2}$ at the $\Gamma$ point is higher than $E_{F}$ in $h \mathrm{VS}_{2} / \mathrm{MoS}_{2}-\mathrm{AB}$ [see Fig. 4(a)]. Thus, the Schottky barrier height is extended into the "negative" region for comparison. Obviously, a similar trend is found in the two systems: $\Phi_{\mathrm{B}, \mathrm{p}}$ decreases monotonously with increasing $P$. Also, the changes are reversible, i.e., the structures relax back to the equilibrium with larger $\Phi_{\mathrm{B}, \mathrm{p}}$ in the absence of pressure, making the systems potentially applicable in sensors. Pressure dependent $p$-type Schottky barrier heights, from another point of view, also constitute an effective strategy to control the degree of $p$-type doping in semiconducting TMDC monolayers. The present $p$-type doping together with the $n$-type doping found in $\mathrm{MoS}_{2}$ on transition metal substrates ${ }^{30,31}$ opens access to $p-n$ junctions.

In order to better understand the metal-semiconductor junctions, we address the plane-averaged charge density difference $\Delta \rho(z)$ to visualize the charge redistribution at the interface. The case of $h \mathrm{VS}_{2} / \mathrm{MoS}_{2}-\mathrm{AB}$ at the equilibrium separation is shown in Fig. 5(a); similar plots are found for the other studied systems. It can be seen that electrons are transferred from the semiconductor side to the interface and the $\mathrm{VS}_{2}$ side, consistent with the results of $p$-type doping in $\mathrm{MoS}_{2}$. The induced charge transfer, which is estimated by integrating $\Delta \rho(z)$, amounts to $0.019|e|$. Particularly, a charge accumulation can be seen around the $\mathrm{V}$ layer, yielding a smaller local magnetic moment with respect to pristine $h \mathrm{VS}_{2}\left(0.92\right.$ versus $\left.1.02 \mu_{\mathrm{B}}\right)$. Figure 5(b) shows the separation dependent charge transfer
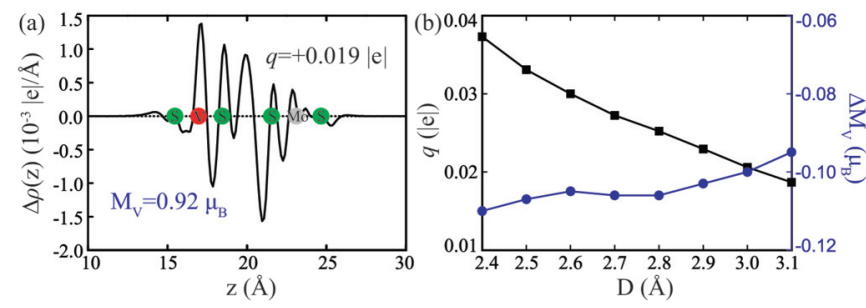

FIG. 5. (Color online) (a) Plane-averaged difference of the electron density, $\Delta \rho(z)$, for $h \mathrm{VS}_{2} / \mathrm{MoS}_{2}-\mathrm{AB}$ at the equilibrium position. The positions of the atoms are indicated, $q$ is the charge transfer, and $M_{V}$ is the magnetic moment of the $\mathrm{V}$ atom. (b) $q$ (black) and $M_{V}$ variation $\left(\Delta M_{V}\right.$, blue) as a function of the interface separation in $h \mathrm{VS}_{2} / \mathrm{MoS}_{2}-\mathrm{AB}$.

and V magnetic moment. When the separation increases, both decrease gradually. This implies that the layer-layer interaction does not preclude charge transfer at the interface, similar to findings for $\mathrm{MoS}_{2} / \mathrm{Ti}_{2} \mathrm{CY}_{2} .{ }^{47}$ However, the charge transfer here is the opposite direction, so that an integration of $\mathrm{MoS}_{2} / \mathrm{VS}_{2}$ and $\mathrm{MoS}_{2} / \mathrm{Ti}_{2} \mathrm{CY}_{2}$ would give rise to a coherent dopant-free all-2D $p-n$ junction. ${ }^{28,50}$

A schematic model is introduced in Fig. 6(a) to explain the effects on the $p$-type Schottky barrier height, which can be written as ${ }^{51}$

$$
\Phi_{B, p}=I_{\mathrm{MoS}_{2}}-\Phi_{\mathrm{VS}_{2}}-\mu_{\mathrm{IS}},
$$

where $I_{\mathrm{MoS}_{2}}$ and $\Phi_{\mathrm{VS}_{2}}$ are the electronic ionization potential of $\mathrm{MoS}_{2}$ and the work function of $\mathrm{VS}_{2}$, respectively. At the contact of $\mathrm{MoS}_{2}$ and $\mathrm{VS}_{2}$ an interface dipole $\mu_{\mathrm{IS}}$ occurs as a result of charge rearrangements. Its sign is negative, since the charge is transferred away from the semiconductor side. Additionally, due to the interaction between $\Gamma$ point states, ${ }^{49}$ the VMB of $\mathrm{MoS}_{2}$ shifts upwards by $\Delta$ from its original position. Note that the semiconducting nature of $\mathrm{MoS}_{2}$ (or $\mathrm{WS}_{2}$ ) is preserved. The modification of the $\mathrm{VS}_{2}$ work function is found to be tiny, i.e., $\Phi_{\mathrm{VS}_{2}} \approx \Phi_{\mathrm{VS}_{2}}^{0}$. As a consequence, Eq. (2) can be rewritten as

$$
\begin{aligned}
\Phi_{B, p} & =\left(I_{\mathrm{MoS}_{2}}^{0}-\Delta\right)-\Phi_{\mathrm{vs}_{2}}^{0}-\mu_{\mathrm{IS}} \\
& =\left(I_{\mathrm{MoS}_{2}}^{0}-\Phi_{\mathrm{vs}_{2}}^{0}\right)-\Delta-\mu_{\mathrm{IS}},
\end{aligned}
$$

where the superscript " 0 " denotes the pristine systems. Data obtained from Eq. (3) are shown by lines in Fig. 6(b) and reproduce the results of the band structure calculations very well. This fact suggests that three factors together determine the Schottky barrier height. The first is the intrinsic (and thus contact) difference between the electronic ionization potentials and work functions, respectively, of the pristine semiconductor and metal monolayers. The second is the $\mathrm{MoS}_{2} / \mathrm{VS}_{2}$ interface interaction, which leads to $\mathrm{MoS}_{2}$ VBM upshifts. Note that, according to Ref. 47, even a large dipole shifts the energy levels only slightly. The last is the charge redistribution induced interface dipole. The interface barrier and $\mathrm{MoS}_{2}$ VBM upshift as a function of the separation are shown in Figs. 6(c) and 6(d), respectively. It can be seen that the latter two factors are larger in $h \mathrm{VS}_{2} / \mathrm{MoS}_{2}-\mathrm{AB}$, suggesting a stronger interaction and more pronounced charge transfer. The third factor depends less on $D$ due to its long-range nature. The ionization potential of 
(a)
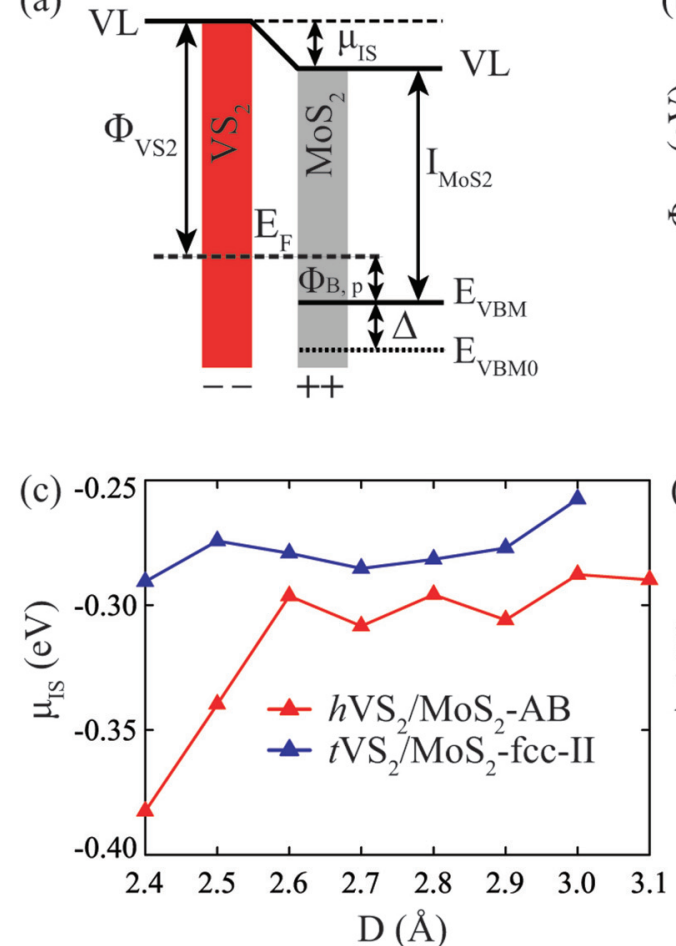
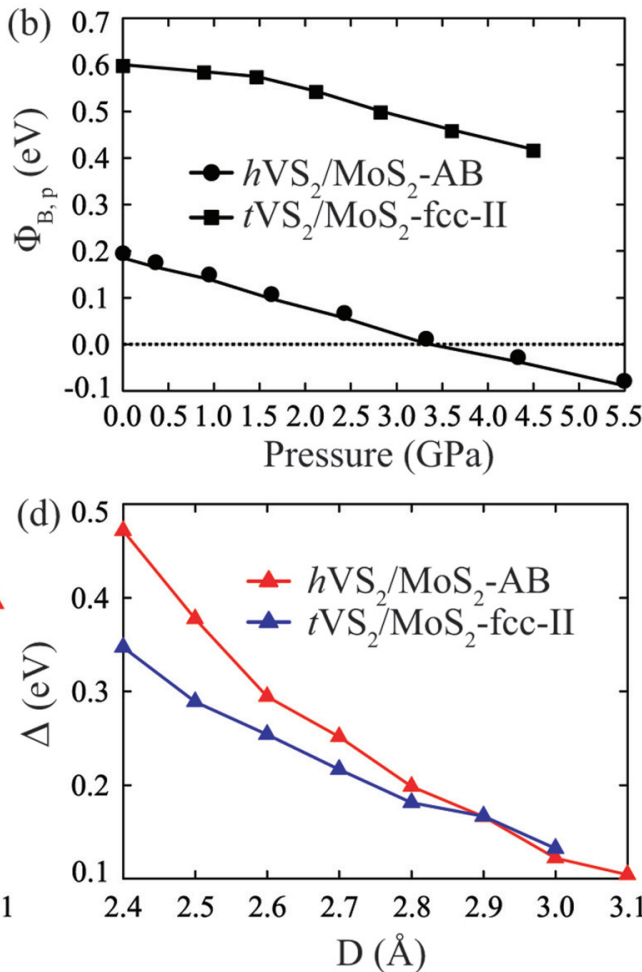

FIG. 6. (Color online) (a) Schematic diagram of the Schottky barrier formation. (b) Schottky barrier height as a function of the vertical compressive pressure in $h \mathrm{VS}_{2} / \mathrm{MoS}_{2}-\mathrm{AB}$ (circles) and $t \mathrm{VS}_{2} / \mathrm{MoS}_{2}$-fcc-II (squares). The lines represent the results obtained from Eq. (3). (c) Interface dipole $\left(\mu_{\mathrm{IS}}\right)$ and (d) $\operatorname{MoS}_{2} \operatorname{VBM}$ upshift $(\Delta)$ as a function of $D$ from $2.4 \AA$ to the respective equilibrium positions.

pristine $\mathrm{MoS}_{2}$ is $5.88 \mathrm{eV}$, giving the first contribution a value of $0.01 \mathrm{eV}$ in $h \mathrm{VS}_{2} / \mathrm{MoS}_{2}-\mathrm{AB}$. Accordingly, $\Phi_{\mathrm{B}, \mathrm{p}}$ is determined by the latter two contributions, where the second is larger than the third for pressure larger than $3.4 \mathrm{GPa}$, leading to negative barrier heights. In $t \mathrm{VS}_{2} / \mathrm{MoS}_{2}$ the first contribution $(0.47 \mathrm{eV})$ is dominant and the latter two almost cancel out each other, thus resulting in higher barriers; see Fig. 6(b). By the similarity of this picture with TMDC/TMDC bilayers, the analytical model can be expected to be applicable to all TMDC metal/semiconductor contacts.

Understanding the spin injection into semiconducting 2D materials is important for spintronics applications. Figure 4(a) demonstrates $100 \%$ spin polarization in $\mathrm{MoS}_{2}$ at $E_{F}$ with one spin channel crossing $E_{F}$ while the other stays below at $D=2.4 \AA(P=5.5 \mathrm{GPa})$ in $h \mathrm{VS}_{2} / \mathrm{MoS}_{2}-\mathrm{AB}$. Similar results are obtained for $h \mathrm{VS}_{2} / \mathrm{WS}_{2}-\mathrm{AB}$. The results imply that it is feasible to achieve $100 \%$ spin polarization in an appropriate pressure range. According to Fig. 6(b), the minimum pressure for which the VBM at the $\Gamma$ point develops a spin splitting and one spin channel crosses $E_{F}$ is around 3.4 GPa. The maximum pressure at which the metallic feature becomes dominant in $\mathrm{MoS}_{2}$, as is the case in $h \mathrm{VS}_{2} / \mathrm{MoS}_{2}$-AA for $D=2.4 \AA$ [see Fig. 3(a)], is calculated to be around $18 \mathrm{GPa}$. Additionally, when the pressure increases from 3.4 to $18 \mathrm{GPa}$ the spin splitting becomes larger and finally a value of $0.70 \mathrm{eV}$ is obtained. However, in $t \mathrm{VS}_{2} / \mathrm{MoS}_{2}$, due to the large difference $(0.47 \mathrm{eV})$ between the ionization potential of pristine $\mathrm{MoS}_{2}$ and the work function of $t \mathrm{VS}_{2}$, the two spin channels at the VBM of $\mathrm{MoS}_{2}$ in the hybrid system hardly will shift above $E_{F}$, making it unlikely to have the same result as in $t \mathrm{VS}_{2} /$ $\mathrm{MoS}_{2}$. Consequently, contacting $\mathrm{MoS}_{2}$ with $h \mathrm{VS}_{2}$ is a good strategy to realize an efficient spin injection for spintronics applications.

\section{CONCLUSIONS}

We have discussed the results of first-principles calculations for the prototypical $\mathrm{VS}_{2} / \mathrm{MoS}_{2}$ and $\mathrm{VS}_{2} / \mathrm{WS}_{2}$ contacts to explore the physics of metal/semiconductor contacts constructed of TMDC monolayers. The degree of $p$-type doping as well as the Schottky barrier height in $\mathrm{MoS}_{2}$ and $\mathrm{WS}_{2}$ upon interface formation can be readily tuned by vertical compressive pressure. In addition, an analytical model has been introduced to understand which quantities determine the barrier height and to which degree. An almost ideal accuracy of the model suggests that three factors play the key role: the difference between the electronic ionization potential of the semiconductor and the work function of the metal, the VBM upshift, and the interface dipole. Moreover, similarity with the interaction in TMDC/TMDC bilayer systems indicates that the model is of general validity for TMDC metal/semiconductor contacts.

As a consequence of magnetic proximity effects, $100 \%$ spin polarization at $E_{F}$ is achieved in the $\mathrm{MoS}_{2}$ layer in the pressure range from approximately 3.4 to $18 \mathrm{GPa}$. Within this range the spin splitting increases up to $0.70 \mathrm{eV}$. The obtained insights into 2D TMDC contacts are significant for designing all-2D metal/semiconductor contacts and also for further spin transport explorations.

\section{ACKNOWLEDGMENT}

This work was supported by a KAUST CRG grant and computational resources were provided by KAUST HPC. 
*Author to whom correspondence should be addressed: yingchun.cheng@kaust.edu.sa; udo.schwingenschlogl@kaust.edu.sa ${ }^{1}$ C. Lee, Q. Li, W. Kalb, X.-Z. Liu, H. Berger, R. W. Carpick, and J. Hone, Science 328, 76 (2010).

${ }^{2}$ F. Schwierz, Nat. Nanotechnol. 5, 487 (2010).

${ }^{3}$ L. Britnell, R. V. Gorbachev, R. Jalil, B. D. Belle, F. Schedin, A. Mishchenko, T. Georgiou, M. I. Katsnelson, L. Eaves, S. V. Morozov, N. M. R. Peres, J. Leist, A. K. Geim, K. S. Novoselov, and L. A. Ponomarenko, Science 335, 947 (2012).

${ }^{4}$ B. Radisavljevic, A. Radenovic, J. Brivio, V. Giacometti, and A. Kis, Nat. Nanotechnol. 6, 147 (2011).

${ }^{5}$ Z. Y. Zhu, Y. C. Cheng, and U. Schwingenschlögl, Phys. Rev. B 84, 153402 (2011).

${ }^{6}$ D. Xiao, G.-B. Liu, W. Feng, X. Xu, and W. Yao, Phys. Rev. Lett. 108, 196802 (2012).

${ }^{7}$ P. Johari and V. B. Shenoy, ACS Nano 6, 5449 (2012).

${ }^{8}$ Y. Y. Hui, X. Liu, W. Jie, N. Y. Chan, J. Hao, Y.-T. Hsu, L.-J. Li, W. Guo, and S. P. Lau, ACS Nano 7, 7126 (2013).

${ }^{9}$ K. F. Mak, C. Lee, J. Hone, J. Shan, and T. F. Heinz, Phys. Rev. Lett. 105, 136805 (2010).

${ }^{10}$ Z. Yin, H. Li, H. Li, L. Jiang, Y. Shi, Y. Sun, G. Lu, Q. Zhang, X. Chen, and H. Zhang, ACS Nano 6, 74 (2012).

${ }^{11}$ K. F. Mak, K. He, C. Lee, G. H. Lee, J. Hone, T. F. Heinz, and J. Shan, Nat. Mater. 12, 207 (2013).

${ }^{12}$ P. Johari and V. B. Shenoy, ACS Nano 5, 5903 (2011).

${ }^{13}$ S. Bertolazzi, J. Brivio, and A. Kis, ACS Nano 5, 9703 (2011).

${ }^{14} \mathrm{~J}$. Pu, Y. Yomogida, K.-K. Liu, L.-J. Li, Y. Iwasa, and T. Takenobu, Nano Lett. 12, 4013 (2012).

${ }^{15}$ A. Castellanos-Gomez, M. Poot, G. A. Steele, H. S. J. van der Zant, N. Agrait, and G. Rubio-Bollinger, Adv. Mater. 24, 772 (2012).

${ }^{16}$ M. A. Lukowski, A. S. Daniel, F. Meng, A. Forticaux, L. Li, and S. Jin, J. Am. Chem. Soc. 135, 10274 (2013).

${ }^{17}$ D. Voiry, H. Yamaguchi, J. Li, R. Silva, D. C. B. Alves, T. Fujita, M. Chen, T. Asefa, V. B. Shenoy, G. Eda, and M. Chhowalla, Nat. Mater. 12, 850 (2013).

${ }^{18}$ X. Huang, Z. Zeng, S. Bao, M. Wang, X. Qi, Z. Fan, and H. Zhang, Nat. Commun. 4, 1444 (2013).

${ }^{19}$ M. Chhowalla, H. S. Shin, G. Eda, L.-J. Li, K. P. Loh, and H. Zhang, Nat. Chem. 5, 263 (2013).

${ }^{20}$ A. Ramasubramaniam, D. Naveh, and E. Towe, Phys. Rev. B 84, 205325 (2011).

${ }^{21}$ S. Bhattacharyya and A. K. Singh, Phys. Rev. B 86, 075454 (2012).

${ }^{22}$ B. Radisavljevic, M. B. Whitwick, and A. Kis, ACS Nano 5, 9934 (2011).

${ }^{23}$ K. Chang and W. Chen, Chem. Commun. 47, 4252 (2011).

${ }^{24}$ K. Chang and W. Chen, ACS Nano 5, 4720 (2011).

${ }^{25}$ Y. Li, H. Wang, L. Xie, Y. Liang, G. Hong, and H. Dai, J. Am. Chem. Soc. 133, 7296 (2011).

${ }^{26}$ W. Zhang, C.-P. Chuu, J.-K. Huang, C.-H. Chen, M.-L. Tsai, Y.-H. Chang, C.-T. Liang, J.-H. He, M.-Y. Chou, and L.-J. Li, arXiv:1302.1230.
${ }^{27}$ J. N. Coleman, M. Lotya, A. O’Neill, S. D. Bergin, P. J. King, U. Khan, K. Young, A. Gaucher, S. De, R. J. Smith, I. V. Shvets, S. K. Arora, G. Stanton, H.-Y. Kim, K. Lee, G. T. Kim, G. S. Duesberg, T. Hallam, J. J. Boland, J. J. Wang, J. F. Donegan, J. C. Grunlan, G. Moriarty, A. Shmeliov, R. J. Nicholls, J. M. Perkins, E. M. Grieveson, K. Theuwissen, D. W. McComb, P. D. Nellist, and V. Nicolosi, Science 331, 568 (2011).

${ }^{28}$ Q. H. Wang, K. Kalantar-Zadeh, A. Kis, J. N. Coleman, and M. S. Strano, Nat. Nanotechnol. 7, 699 (2012).

${ }^{29}$ I. Popov, G. Seifert, and D. Tománek, Phys. Rev. Lett. 108, 156802 (2012).

${ }^{30}$ W. Chen, E. J. G. Santos, W. Zhu, E. Kaxiras, and Z. Zhang, Nano Lett. 13, 509 (2013).

${ }^{31}$ J.-R. Chen, P. M. Odenthal, A. G. Swartz, G. C. Floyd, H. Wen, K. Y. Luo, and R. K. Kawakami, Nano Lett. 13, 3106 (2013).

${ }^{32}$ E. I. Rashba, Phys. Rev. B 62, R16267 (2000).

${ }^{33}$ G. Schmidt, D. Ferrand, L. W. Molenkamp, A. T. Filip, and B. J. van Wees, Phys. Rev. B 62, R4790 (2000).

${ }^{34}$ Y. C. Cheng, Z. Y. Zhu, W. B. Mi, Z. B. Guo, and U. Schwingenschlögl, Phys. Rev. B 87, 100401 (2013).

${ }^{35}$ Y. Ma, Y. Dai, M. Guo, C. Niu, Y. Zhu, and B. Huang, ACS Nano 6, 1695 (2012).

${ }^{36}$ Y. Zhou, Z. Wang, P. Yang, X. Zu, L. Yang, X. Sun, and F. Gao, ACS Nano 6, 9727 (2012).

${ }^{37}$ J. Feng, X. Sun, C. Wu, L. Peng, C. Lin, S. Hu, J. Yang, and Y. Xie, J. Am. Chem. Soc. 133, 17832 (2011).

${ }^{38}$ K. Xu, P. Chen, X. Li, C. Wu, Y. Guo, J. Zhao, X. Wu, and Y. Xie, Angew. Chem. Int. Ed., doi:10.1002/anie.201304337.

${ }^{39}$ C. Ataca, H. Şahin, and S. Ciraci, J. Phys. Chem. C 116, 8983 (2012).

${ }^{40}$ H. Haugen, D. Huertas-Hernando, and A. Brataas, Phys. Rev. B 77, 115406 (2008).

${ }^{41}$ D. Liu, Y. Hu, H. Guo, and X. F. Han, Phys. Rev. B 78, 193307 (2008).

${ }^{42}$ H. X. Yang, A. Hallal, D. Terrade, X. Waintal, S. Roche, and M. Chshiev, Phys. Rev. Lett. 110, 046603 (2013).

${ }^{43}$ T. Bucko, J. Hafner, S. Lebégue, and J. G. Ángyán, J. Phys. Chem. A 114, 11814 (2010).

${ }^{44}$ S. Grimme, J. Comput. Chem. 27, 1787 (2006).

${ }^{45}$ H. Zhang, L.-M. Liu, and W.-M. Lau, J. Mater. Chem. A 1, 10821 (2013).

${ }^{46}$ C. S. Rout, B.-H. Kim, X. Xu, J. Yang, H. Y. Jeong, D. Odkhuu, N. Park, J. Cho, and H. S. Shin, J. Am. Chem. Soc. 135, 8720 (2013).

${ }^{47}$ L.-Y. Gan, Y.-J. Zhao, D. Huang, and U. Schwingenschlögl, Phys. Rev. B 87, 245307 (2013).

${ }^{48}$ J. Tersoff, Phys. Rev. Lett. 52, 465 (1984).

${ }^{49}$ H.-P. Komsa and A. V. Krasheninnikov, Phys. Rev. B 88, 085318 (2013).

${ }^{50}$ G. Eda, T. Fujita, H. Yamaguchi, D. Voiry, M. Chen, and M. Chhowalla, ACS Nano 6, 7311 (2012).

${ }^{51}$ R. T. Tung, Phys. Rev. Lett. 84, 6078 (2000). 